\title{
Optical modeling of four Fresnel-based high-CPV units
}

\section{Juan P. Ferrer-Rodríguez ${ }^{\mathrm{a}, *}$, Hasan Baig ${ }^{\mathrm{b}}$, Eduardo F. Fernández ${ }^{\mathrm{a}}$, Florencia Almonacid ${ }^{\mathrm{a}}$, Tapas Mallick ${ }^{\mathrm{b}}$, Pedro Pérez-Higueras ${ }^{b}$}

${ }^{a}$ IDEA Solar Research Group, Center for Advanced Studies in Energy and Environment (CEAEMA), Universidad de Jaén, Las Lagunillas Campus, Jaén 23071, Spain

${ }^{\mathrm{b}}$ Environment and Sustainability Institute, University of Exeter, Penryn Campus, Penryn TR10 9FE, United Kingdom

\section{A R T I C L E I N F O}

Article history:

Received 7 April 2017

Received in revised form 21 June 2017

Accepted 6 July 2017

Available online 14 July 2017

\section{Keywords:}

High concentrator photovoltaics

Optical simulations

\begin{abstract}
A B S T R A C T
High Concentrator Photovoltaic (HCPV) units are typically based on the use of Fresnel lenses, refractive secondary optical elements (SOE), and triple-junction (TJ) solar cells. In this work, a detailed optical modeling is applied to analyze the performance of four Fresnel-based HCPV units equipped with different refractive SOEs while considering the subcells current density generation. Wavelength-dependent material properties are utilized while simulating the optical performance. The spectral response of a typical TJ solar cell is also included. This modeling allows to establish the subcell current limitation and the spectral matching ratio, SMR, values in each case. The following SOEs have been used for simulating the HCPV units: (i) Dielectric-cross compound-parabolic-concentrator (DCCPC), (ii) (SIngle-Lens-Optical element) SILO-Pyramid, (iii) Refractive truncated pyramid (RTP) and, (iv) Trumpet. Results show that the HCPV units with SOEs RTP and Trumpet, exhibit bottom subcell current limitation and lowest optical polychromatic efficiency, this is partly due to the irradiance absorption in the bottom cell spectral region and longer optical path length of the concentrated rays within the SOE material. In the case of the HCPV unit with the DCCPC SOE, top and bottom subcells limit the current generation alternatively depending on the misalignment angle of the HCPV unit respect to the simulated sunrays. None of the SMR parameters are equal to 1 under normal alignment of the HCPV units. The short-circuit current density distributions for each subcell in each case are studied under normal alignment and under $1^{\circ}$ of misalignment angle.
\end{abstract}

(ㄷ) 2017 Elsevier Ltd. All rights reserved.

\section{Introduction}

Fresnel-based High Concentrator Photovoltaic (HCPV) devices are typically equipped with secondary optical elements (SOE) in order to improve their performance, since for increased concentrations, the tolerance to misalignment decreases (Pérez-Higueras and Fernández, 2015; Kumar et al., 2015). Some authors (Victoria et al., 2009; Fu et al., 2010; Benitez et al., 2010) have already pointed the advantages of using SOEs, which can be summarized as the increase of: (i) the optical efficiency by collecting more concentrated sunrays, (ii) the acceptance angle, which enhances the energy production and decreases manufacturing, installation and tracker costs, and (iii) the spatial and spectral irradiance uniformity over the multi-junction (MJ) solar cell (Fu et al., 2010). The non-uniform illumination is one of the key issues of the concentrator systems (Victoria et al., 2009; Baig et al., 2012), since the production and collection of the generated current may be altered depending on how uniformly the light is concentrated.

\footnotetext{
* Corresponding author.

E-mail address: jferrer@ujaen.es (J.P. Ferrer-Rodríguez).
}

The literature shows detailed studies analyzing the performance of different SOEs under the same primary optical element (POE). Victoria et al. (2009) compared some different SOEs under the same circular plano-convex aspheric lens, results showed that the refractive compound parabolic concentrator (CPC) is more efficient and has a wider acceptance angle than those SOEs based on the SILO (SIngle-Lens-Optical element) design (James, 1989), while, on the other hand, the refractive CPC showed much less irradiance uniformity over the solar cell. Fu et al. (2010) compared three different refractive SOEs made of BK7 or B270 glass under the same Fresnel POE with a geometrical concentration of $800 \times$ : kaleidoscope, half-egg and domed kaleidoscope with breaking-symmetry top. They found that the domed kaleidoscope had a better performance, greater irradiance uniformity and acceptance angle. Miñano et al. (2013) presented some free-form Fresnel-Köhler SOE designs achieving relative good irradiance uniformity while avoiding total internal reflection (TIR), and therefore, light leakage between the solar cell and the SOE. Chen and Chiang (2015) showed a "kaleidoscope with equal optical path design (KOD)" SOE based on an ellipsoidal-top, a middle conic section and a bottom region that works under TIR and ends at a square surface. They 
compared the KOD design with other SOEs like the refractive truncated pyramid (RTP), showing higher acceptance angle. The above commented studies are very interesting from both a scientific and an industrial point of view, nevertheless, the refractive index wavelength dependency of the materials simulated is not included in the ray tracing in those works, excepting in that of Chen and Chiang (2015). Moreover, no spectral light absorption inside the materials was simulated or the spectral response of the MJ cell was considered. Among the different studies, only one, in which those last aspects were considered and simulated, has been found. This is the case of Espinet-González et al. (2012), who simulated and compared some refractive SOE, although not under the same POE. They took into account both absorption coefficient and refractive index as a function of the wavelength, and even of the spectral response of the MJ cell, however no related material property data were presented. They showed spatial profiles of the photocurrent density for each subcell, and found the illumination from Fresnel-Köhler design to be the most uniform in comparison to the SILO and the RTP.

An optical modeling intended to improve the approach to real optical phenomena is implemented in this work, including key features, like: (i) standard terrestrial spectrum, (ii) angular distribution of sunrays, (iii) Fresnel POE, (iv) wavelength dependency of refractive index and (v) absorption coefficient for both POE and SOE and for ( $v$ ) absorption coefficient, and (vi) spectral response of each subcell within the TJ solar cell. This modeling provides the simulated subcell short-circuit currents of a typical TJ solar cell. This allows to know which subcell is limiting the current generation and to properly calculate the optical polychromatic efficiency and spectral matching ratio parameters. These calculations are conducted for normal alignment of the HCPV unit respect to the sunrays and also by different misalignment angles. Moreover, using these results, instead of analyzing the irradiance uniformity on the TJ solar cell, the short-circuit current density uniformity is analyzed for each subcell and for all the misalignment angles.

This modeling is applied in this work to four different Fresnelbased HCPV units equipped with refractive SOEs. These SOEs are designed and their performance analyzed through ray tracing simulations. The materials chosen for the POE and all the SOEs is poly (methyl methacrylate), PMMA, due to its ease of fabrication in a future experimental validation. Both a typical Fresnel lens and a typical triple-junction (TJ) concentrator solar cell are maintained constant for all of the HCPV units while using different SOEs. The different SOEs designed include: (i) Dielectric-Cross CompoundParabolic-Concentrator (DCCPC), (ii) SILO-Pyramid, (iii) RTP, and (iv) Trumpet.

\section{Description of the HCPV units simulated}

In this section, the elements configuring the four HCPV units simulated are described. Firstly, the common elements to all the HCPV units, i.e. Fresnel lens POE and TJ solar cell, are detailed. Secondly, the four different SOEs composing the HCPV units are described and briefly explained.

\subsection{Primary optics and the TJ solar cell}

The primary optical element (POE) used in this study is a typical square Fresnel lens made of PMMA with an effective area of $130 \times 130 \mathrm{~mm}^{2}$ and a focal distance of $152 \mathrm{~mm}$. Its thickness is $1.8 \mathrm{~mm}$ and the ring facet spacing is $0.381 \mathrm{~mm}$. F-number is equal to 0.83 and the geometrical concentration ratio between the areas of POE and TJ solar cell, $C_{g}=A_{\text {lens }} / A_{\text {cell }}$, is around $559 \times$, since the TJ solar cell is a square of $5.5 \mathrm{~mm}$ side. These parameters defining the POE are listed in Table 1.
Table 1

Summary of parameters defining the Fresnel lens simulated.

\begin{tabular}{ll}
\hline Parameter & Value \\
\hline Size $\left[\mathrm{mm}^{2}\right]$ & $130 \times 130$ \\
Focal distance [mm] & 152.0 \\
F-Number [-] & 0.83 \\
Facet spacing [mm] & 0.381 \\
Thickness [mm] & 1.8 \\
\hline
\end{tabular}

The solar cell is a typical TJ solar cell made of GaInP/GaInAs/Ge on Ge substrate. It is simulated through the spectral response characteristic of each subcell, top, middle ("mid") and bottom ("bot"), as plotted in Fig. 5 (Section 3). The short-circuit current density values under STC (standard test conditions, AM1.5D, ASTM G173-03, $1000 \mathrm{~W} / \mathrm{m}^{2}, 25^{\circ} \mathrm{C}$ ) are simulated to be (Anon., n.d.): $J_{\text {sc,top }}{ }^{\text {sun }}=15.6 \mathrm{~mA} / \mathrm{cm}^{2} ; \quad J_{s c, \text { mid }}{ }^{1 \text { sun }}=15.7 \mathrm{~mA} / \mathrm{cm}^{2} ; \quad J_{s c, \text { bot }}{ }^{1 \text { sun }}=$ $19.2 \mathrm{~mA} / \mathrm{cm}^{2}$. Whereas for the whole device, $J_{s c}{ }^{1 \text { sun }}=15.6 \mathrm{~mA} / \mathrm{cm}^{2}$, since the subcells are series connected. Table 2 shows the main parameters of the TJ solar cell simulated.

\subsection{Four different SOEs}

The different SOEs under study are shown in Fig. 1. Parametric optimization is carried out by varying their heights/truncation to reach a trade-off between efficiency and their acceptance angle. Fig. 1 shows a rendering of the four SOEs obtained: (a) DCCPC, (b) SILO-Pyramid, (c) RTP, and (d) Trumpet.

In order to have an idea of the relative SOE sizes to scale, both height and the shape of each SOE are shown in Fig. 2:

For each HCPV unit, the SOE entrance surface is located at the focal distance respect to the POE except for the case of the SILOPyramid, which is located $2 \mathrm{~mm}$ closer to the POE in order to improve its performance. In the following lines, the different SOEs are explained.

The DCCPC SOE is based on the geometrical principles of the $\mathrm{CPC}$ but combining both square entrance and exit surfaces (O'Gallagher, 2008; Cruz-Silva et al., 2016; Cooper et al., 2013). Its exit aperture matches the TJ solar cell area, like in all the SOEs here investigated. The DCCPC SOE here designed is defined by a characteristic axis tilt of the parabola, $\theta_{c}=32^{\circ}$, with a focal distance of $f=4.2073 \mathrm{~mm}$ and a height $h=11 \mathrm{~mm}$. $\theta_{c}$ is close to the maximum angular size of the POE seen from its focal point. The SILOPyramid SOE corresponds to a modification of the bottom part of the standard SILO (also called “Dome-B" (Victoria et al., 2009)), which is calculated applying the Fermat's Principle. This modification takes advantage of the TIR at the walls of the pyramid. The resulting SILO-Pyramid SOE has a total height of $10.54 \mathrm{~mm}$, whereas the truncated pyramid bottom part has a height of $3.2 \mathrm{~mm}$ and a total virtual entrance square of $10 \mathrm{~mm}$ side. The RTP SOE is based on a statistical design approach, as Fu et al. described (Fu et al., 2010), and can be also utilized as homogenizer in a Cassegrain-type concentrator (Shanks et al., 2016, 2017). The resulting design has a height of $17 \mathrm{~mm}$ with a square entrance of $12 \mathrm{~mm}$ side. The Trumpet SOE here designed is based on the use

Table 2

Summary of parameters defining the triple-junction solar cell simulated.

\begin{tabular}{ll}
\hline Parameter & Value \\
\hline Size $\left[\mathrm{mm}^{2}\right]$ & $5.5 \times 5.5$ \\
Materials (top, mid, bot) & GaInP/GaInAs/Ge \\
$J_{\text {sc,top }{ }^{1 \text { sun }}\left[\mathrm{mA} / \mathrm{cm}^{2}\right]}{ }^{1 \operatorname{sun}}\left[\mathrm{mA} / \mathrm{cm}^{2}\right]$ & 15.6 \\
$J_{\text {sc, mid }}{ }^{\operatorname{sun}}\left[\mathrm{mA} / \mathrm{cm}^{2}\right]$ & 15.7 \\
$J_{\text {sc, bot }}{ }^{2}[\mathrm{~cm}$ \\
\hline
\end{tabular}




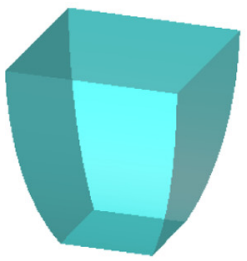

(a)

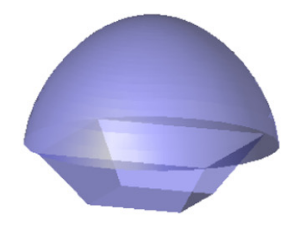

(b)

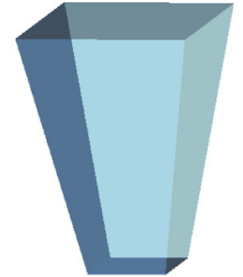

(c)

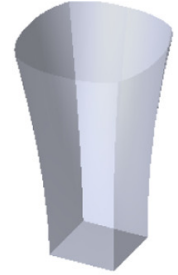

(d)

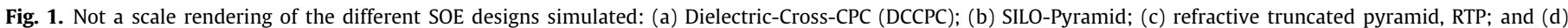
Trumpet.

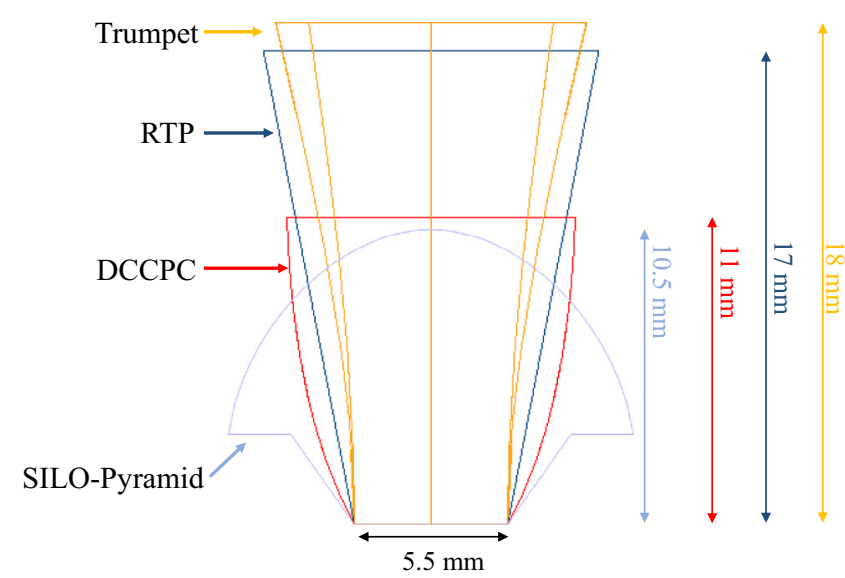

Fig. 2. Layout of the different SOEs profiles to scale with their height specified.

of a total of eight hyperbolic edges (Reddy et al., 2014), four ones for the vertexes of the exit surface and other four ones for the corresponding center of each side. The asymptotes of all of the hyperbolic edges maintain an angle of $15^{\circ}$ respect to the symmetry axis of the SOE. The hyperbolic edges are calculated following the description of O'Gallagher for the design of the Compound Hyperbolic Concentrator (CHC) (O'Gallagher, 2008). The solid volume of the Trumpet is built in a CAD environment (SolidWorks) using the hyperbolic edges as guiding curves until reaching a height of $18 \mathrm{~mm}$. The entrance surface is composed by four arcs of circumference whose centers are almost coincident to the center of the entrance surface.

\section{Optical modeling}

In this section, each feature of the optical modeling implemented in this work is carefully detailed. Moreover, this modeling is compared with other optical modeling works in the literature.

\subsection{Description}

An original and realistic optical modeling has been developed during this study. This optical modeling includes some nonidealities that are not usually taken into account simultaneously in previous research. These considerations include the use of: (i) standard terrestrial spectrum, (ii) angular distribution of sunrays, (iii) Fresnel POE, (iv) wavelength dependency of refractive index and (v) absorption coefficient for both POE and SOE, and (vi) spectral response of each subcell within the TJ solar cell. Moreover, as a result of those characteristics, this optical modeling provides the next output features -which are not available using a simpler optical modeling: (a) subcell short-circuit current generation, (b) spectral matching ratio analysis, (c) optical polychromatic efficiency, (d) effective acceptance angle calculation, and (e) subcell shortcircuit current density uniformity.

\subsection{Optical modeling features}

\section{(i) Standard terrestrial spectrum and (ii) angular distribu- tion of sunrays}

Sunrays are simulated under standard terrestrial direct spectrum ASTM G173-03 within the wavelength range of 0.3 and $2.5 \mu \mathrm{m}$. Also, the angular distribution of sunrays ( $4.7 \mathrm{mrad}$ of angular size) is included in the simulations. See in Fig. 3 both normalized spectra, the one introduced and simulated in the ray tracing software and the standard one, which are almost identical. From here, it will be noted 1 sun $=1000 \mathrm{~W} / \mathrm{m}^{2}$ of standard terrestrial spectrum ASTM G173-03 or, simply, 1 sun of DNI.

\section{(ii) Fresnel POE}

The POE is modeled as an aspheric Fresnel lens using conical facets whose angles are calculated in order to focus the light from a point located at the object distance before the lens, to a point located at the image distance after the lens. The facet angle, $\beta$, is calculated through Eq. (1) (Leutz and Suzuki, 2001):

$\tan \beta=\frac{\sin \theta_{1}+\sin \theta_{2}}{\sqrt{\left(n^{2}-\sin ^{2} \theta_{1}\right)}-\cos \theta_{2}}$

with $\theta_{1}$ the incident angle of rays on the Fresnel lens, $\theta_{2}$ the angle of light exiting the Fresnel lens, and $n$ the refractive index of the material. In this work, the object distance is set infinite and the image distance is $152 \mathrm{~mm}$.

\section{(iii) Wavelength dependency of refractive index for POE and SOE}

As mentioned above, both POE and SOE are modeled to be made of standard PMMA in each HCPV unit. The correspondent material property is defined through a refractive index, $n(\lambda)$, and an absorption coefficient, $\alpha_{P}(\lambda)$, for every wavelength, $\lambda$, in the range or inter-

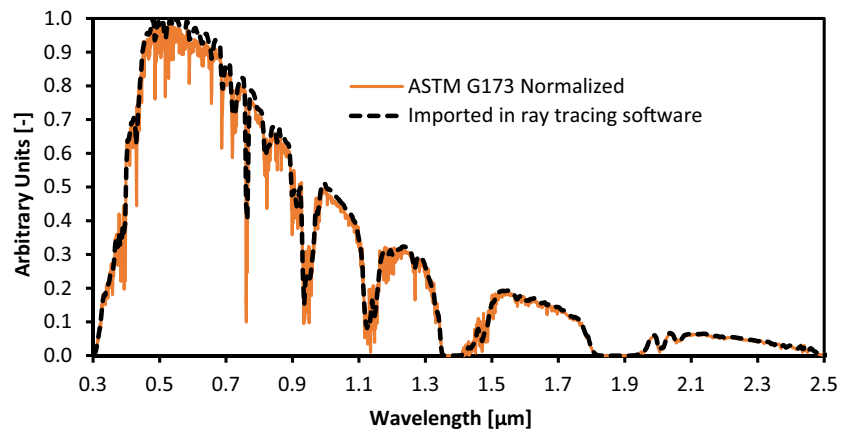

Fig. 3. ASTM G173 and simulated solar spectra, both normalized. 
est, in this case, from 0.3 to $2.5 \mu \mathrm{m}$. Refractive index values are taken from the model of (Beadie et al., 2015) for PMMA and extended as a constant to the wavelength range mentioned (see Fig. 4). According to the aforementioned refractive index data of PMMA, wavelength-dependent Fresnel reflections and chromatic dispersion are simulated in the ray tracing.

\section{(iv) Wavelength dependency of absorption coefficient for POE and SOE}

Concerning the light absorption inside the materials, the BeerLambert law of transmission is applied (see Eq. (2)):

$\Phi_{T}=\Phi_{I} e^{-\alpha_{P} h}$

where $\Phi_{T}$ and $\Phi_{I}$ are the transmitted and incident flux respectively, $h$ is the bulk thickness and $\alpha_{P}$ is the absorption coefficient (for PMMA in our case). Then, the flux absorbed inside the material, $\Phi_{A}$, is (see Eq. (3)):

$\Phi_{A}=\Phi_{I}\left(1-e^{-\alpha_{\mathrm{P}} h}\right)$.

In this work, absorptance data from (Miller et al., 2011) for standard PMMA for a known bulk thickness are used in order to calculate $\alpha_{P}(\lambda)$. The resulting $\alpha_{P}(\lambda)$ values are plotted in Fig. 5 . It can be observed that, approximately, the absorption is negligible in the wavelength region where the middle subcell is active. However, $\alpha_{P}(\lambda)$ is not zero for the whole spectral response region of the TJ solar cell. This may have an impact on the subcell current generated and, consequently, on the performance of each HCPV unit modeled in this work.

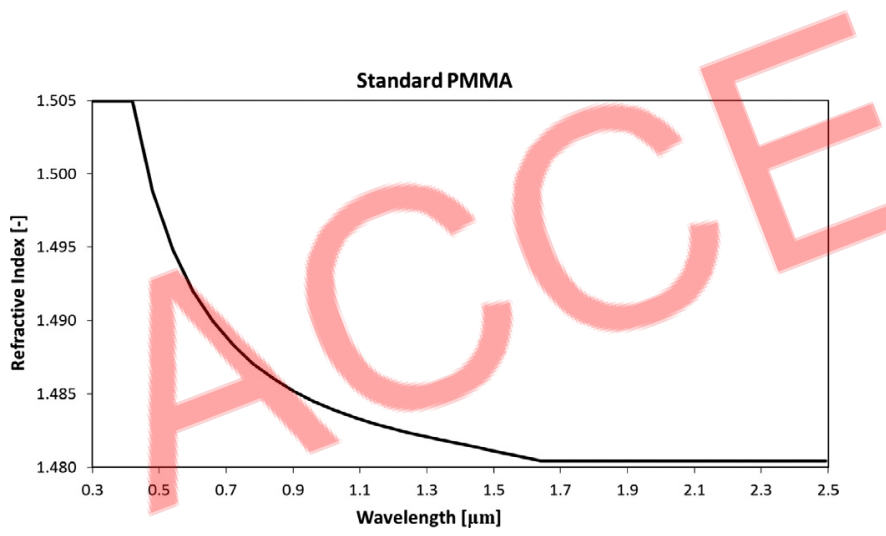

Fig. 4. Refractive index characteristic of standard PMMA used in the simulations.

\section{(v) Spectral response of each subcell within the TJ solar cell}

The TJ solar cell is modeled as composed of three different subcells. Each subcell is simulated separately as a surface with an absorption property according to the external quantum efficiency, $E Q E$, values of each subcell of a typical TJ solar cell. The typical spectral response data of each simulated subcell surface of a TJ solar cell are plotted in Fig. 5. These plotted values correspond to the spectral response data, $S R_{\text {subcell }}(\lambda)$, normalized to the total maximum value of spectral response of the three subcells, which corresponds to around $S R_{\max }=0.977 \mathrm{~A} / \mathrm{W}$.

\subsubsection{Optical modeling output features}

\section{(vi) Subcell short-circuit current density generation}

The utilization of the $S R_{\text {subcell }}(\lambda)$ data allows us to obtain the current density generated, $J_{s c, \text { subcell }}$, for each simulated subcell surface. The expression used for calculating the current density generated by each subcell is given by Eq. (4):

$$
\begin{aligned}
J_{\text {sc,sub-cell }} & =\int S R_{\text {subcell }}(\lambda) \cdot E(\lambda) \cdot d \lambda \\
& =\frac{q}{h \cdot c} \int E Q E_{\text {subcell }}(\lambda) \cdot E(\lambda) \cdot \lambda \cdot d \lambda,
\end{aligned}
$$

where $q$ is the electric charge, $h$ is the Planck's constant, $c$ is the speed of light in vacuum, $E(\lambda)$ is the spectral irradiance impinging on the solar cell simulated through ray tracing, and $\operatorname{EQE}_{\text {subcell }}(\lambda)$ is the external quantum efficiency of each subcell in each case.

\section{(vii) Spectral matching ratio analysis}

The spectral matching ratio, $S M R$, parameters are related to the current density generated in each subcell. For a TJ solar cell, the next SMR parameters can be defined: $S M R$ (top/mid), SMR(top/bot) and $S M R$ (mid/bot). SMR(top/mid) can be defined as (Domínguez et al., 2013):

$S M R($ top $/$ mid $)=\frac{\int_{s c, \text { top }}^{\text {conc }} / J_{s c, \text { top }}^{1 \text { sun }}}{\int_{s c, \text { mid }}^{\text {conc }} / J_{s c, \text { mid }}^{1 s u n}}$

where $J_{s c, \text { subcell }}^{c o n c}$ is the current density generated by the corresponding subcell under concentrated illumination and $J_{\text {sc,subcell }}{ }^{1 \text { sun }}$ is analogous to 1 sun of DNI. Similarly, the other spectral matching ratios, $S M R$ (top/bot) and SMR (mid/bot) can be defined just by replacing the corresponding subcell short-circuit density currents in Eq. (5). SMR

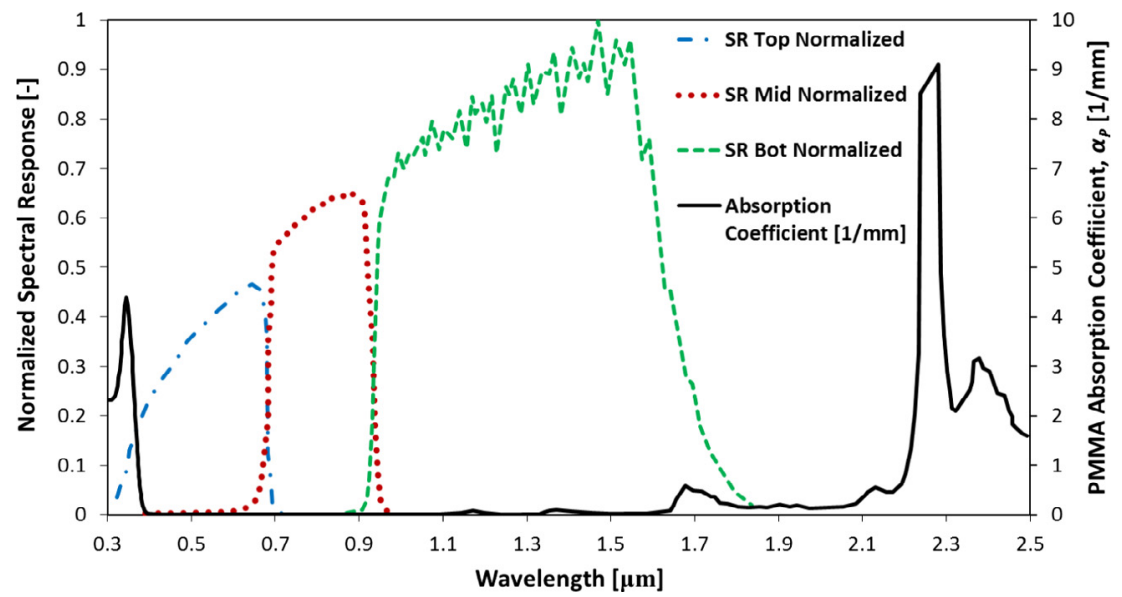

Fig. 5. Absorption coefficient, $\alpha_{P}(\lambda)$, of standard PMMA and normalized spectral response characteristic of each subcell. 
(top $/$ mid $)=1$ means that the proportion between top and mid subcell photocurrents under concentrated irradiance impinging on the TJ solar cell is the same as compared to standard conditions 1 sun of DNI. In this case, the concentrated spectral irradiance is called equivalent to the standard spectrum with respect to the top and mid subcells. $S M R($ top $/$ mid $)<1$ represents a lower irradiance contribution of the top-wavelength region than the midwavelength one of the concentrated sunlight on the TJ solar cell when comparing to the case of the TJ solar cell under 1 sun of DNI, i.e. without any concentrator system. And $\operatorname{SMR}($ top $/$ mid $)>1$ means vice versa. This reasoning is extended to $\operatorname{SMR}($ top/bot) and $S M R$ (mid/bot) by changing the correspondent short-circuit current densities(Rodrigo et al., 2017). The study of these indexes is interesting due to the strong spectral dependence of multi-junction CPV systems in outdoor conditions (Fernández et al. 2014, 2016).

\section{(viii) Optical polychromatic efficiency}

Using the $J_{s c, \text { subcell }}$ values obtained through ray tracing, the optical polychromatic efficiency, $\eta_{\text {opt }}$, is calculated through Eq. (6) (Benitez et al., 2010):

$\eta_{\text {opt }}=\frac{J_{s c}^{\text {conc }}}{C_{g} J_{s c}^{1 s u n}}=\frac{\min \left\{J_{s c, \text { top }}^{\text {conc }}, J_{s c, \text { mid }}^{\text {conc }}, J_{s c, \text { bot }}^{\text {conc }}\right\}}{C_{g} \min \left\{J_{s c, \text { top }}^{1 \text { sun }}, J_{s c, \text { mid }}^{1 \text { 1sun }}, J_{s c, \text { bot }}^{1 s u n}\right\}}$.

This definition of the optical efficiency takes into account the series connection of a typical TJ solar cell and how its resulting $J_{s c}$ is obtained. Therefore, it is linked to the solar cell used as target, instead of only considering the optical efficiency as the relation between the output radiant flux over the incoming one to the concentrator system.

\section{(ix) Effective acceptance angle calculation}

The angular performance of the different HCPV units is characterized through the acceptance angle, $\alpha$, where the $\eta_{\text {opt }}$ drops to $90 \%$ respect to the maximum. It is also helpful to use the figure of merit effective concentration-acceptance angle product, CAP*, (Eq. (7)), which cannot be greater than the refractive index of the medium surrounding the TJ solar cell and also takes into account the angular distribution of sunrays (Benitez et al., 2010):

$C A P^{*}=\sqrt{C_{g} \cdot \sin \alpha}$

\section{(xi) Subcell short-circuit current density uniformity}

Instead of analyzing the irradiance distributions over the $\mathrm{TJ}$ solar cell, short-circuit current density generated distributions for each subcell under the concentrated irradiance are obtained and analyzed. The normalized $J_{\text {sc,subcell }}^{\text {conc }}$ spatial distributions for each subcell and for both normal alignment and $1^{\circ}$ of tilt angle are shown in Fig. 9 in Section 4.

\subsection{Optical modeling in the literature versus present study}

Table 3 shows a comparison among different optical modeling works in the literature for concentrator systems that are based on the use of a refractive lens as POE. It can be found that in the majority of the modeling works found, the next features are usually taken into account: (i) standard terrestrial spectrum, (ii) angular distribution of sunrays, (iii) Fresnel POE, (iv) absorption inside dielectric materials and ( $v$ ) effective acceptance angle calculation. Moreover, the next features are only included in some optical modeling works: (i) wavelength dependency of refractive index for POE and SOE, (ii) wavelength dependency of absorption coefficient for POE and SOE, (iii) spectral response of each subcell within the TJ solar cell, (iv) subcell short-circuit current density generation, (v) optical polychromatic efficiency and (vi) subcell short-circuit current density uniformity. The optical modeling utilized in this work, using a ray tracing software (TracePro), includes all of those features, as can be seen in Table 3. Note that the feature "Absorption inside dielectric materials" is also included since some authors estimate absorption losses without detailing the absorption coefficient of the material.

\section{Results and discussion}

In this section, the numerical results (quantitative) obtained via simulations are deeply analyzed applying a reasoning derived from the different features of the optical modeling. Additionally, some qualitative results are also analyzed, like the short-circuit current density uniformity of the different subcells.

\subsection{Quantitative modeling results}

\subsubsection{Results under normal alignment and $1^{\circ}$ of tilt angle}

As commented previously, ray tracing simulations are conducted for the four Fresnel-based high concentrator units. For each unit, the three subcells of the TJ solar cell are simulated obtaining the different short-circuit current densities. A preliminary collection of simulation results of the four HCPV units under normal alignment respect to the incident sunrays is given in Table 4, which shows the resulting short-circuit current density generated of each subcell, the optical polychromatic efficiency and the spectral matching ratios among the three subcells.

Table 3

Summary of different optical modeling works of lens-POE-based systems in the literature.

\begin{tabular}{|c|c|c|c|c|c|c|}
\hline Feature & $\begin{array}{l}\text { Fu et al. } \\
(2010)\end{array}$ & $\begin{array}{l}\text { Benitez et al. } \\
(2010)\end{array}$ & $\begin{array}{l}\text { Espinet-González et al. } \\
(2012)\end{array}$ & $\begin{array}{l}\text { Miñano et al. } \\
(2013)\end{array}$ & $\begin{array}{l}\text { Chen and Chiang } \\
(2015)\end{array}$ & $\begin{array}{l}\text { This work. } \\
2017\end{array}$ \\
\hline Standard terrestrial spectrum & $\mathrm{X}$ & $\mathrm{X}$ & $\mathrm{X}$ & $\mathrm{X}$ & $\mathrm{X}$ & $\mathrm{X}$ \\
\hline Angular distribution of sunrays & $\mathrm{X}$ & $\mathrm{X}$ & & $\mathrm{X}$ & & $\mathrm{X}$ \\
\hline Fresnel POE & $\mathrm{X}$ & $\mathrm{X}$ & $\mathrm{X}$ & $\mathrm{X}$ & $\mathrm{X}$ & $\mathrm{X}$ \\
\hline $\begin{array}{l}\text { Wavelength dependency of refractive index for POE } \\
\text { and SOE }\end{array}$ & & & $\mathrm{X}$ & & $\mathrm{X}$ & $\mathrm{X}$ \\
\hline Absorption inside dielectric materials & & $\mathrm{X}$ & $\mathrm{X}$ & $\mathrm{X}$ & $\mathrm{X}$ & $\mathrm{X}$ \\
\hline $\begin{array}{l}\text { Wavelength dependency of absorption coefficient } \\
\text { for POE and SOE }\end{array}$ & & & $\mathrm{X}$ & & & $\mathrm{X}$ \\
\hline $\begin{array}{l}\text { Spectral response of each subcell within the TJ solar } \\
\text { cell }\end{array}$ & & $\mathrm{X}$ & $\mathrm{X}$ & & & $\mathrm{X}$ \\
\hline Subcell short-circuit current density generation & & $\mathrm{X}$ & $\mathrm{X}$ & & & $\mathrm{X}$ \\
\hline Spectral matching ratio analysis & & & & & & $\mathrm{X}$ \\
\hline Optical polychromatic efficiency & & $\mathrm{X}$ & & & & $\mathrm{X}$ \\
\hline Effective acceptance angle calculation & $\mathrm{X}$ & $\mathrm{X}$ & & $\mathrm{X}$ & & $\mathrm{X}$ \\
\hline Subcell short-circuit current density uniformity & & & $\mathrm{X}$ & & & $\mathrm{X}$ \\
\hline
\end{tabular}


Table 4

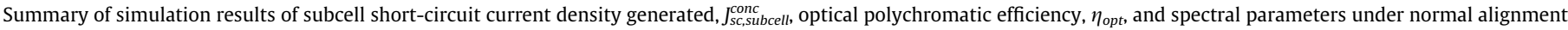
of each HCPV unit respect to the sunrays for each Fresnel-based concentrator unit.

\begin{tabular}{|c|c|c|c|c|}
\hline Parameter & SILO-Pyramid & DCCPC & RTP & Trumpet \\
\hline$J_{s c, \text { top }}^{c o n c}\left[\mathrm{~A} / \mathrm{cm}^{2}\right]$ & 7.29 & 7.30 & 7.31 & 7.29 \\
\hline$J_{s c, \text { mid }}^{\text {conc }}\left[\mathrm{A} / \mathrm{cm}^{2}\right]$ & 7.60 & 7.60 & 7.60 & 7.60 \\
\hline$J_{s c, \text { bot }}^{\text {conc }}\left[\mathrm{A} / \mathrm{cm}^{2}\right]$ & 7.67 & 7.62 & 7.15 & 7.08 \\
\hline Limiting subcell & Top & Top & Bot & Bot \\
\hline$\eta_{\text {opt }}[\%]$ & 83.4 & 83.6 & 81.8 & 81.0 \\
\hline$\eta_{\text {opt }}[\%]$ ideal case & 85.3 & 84.6 & 83.2 & 86.6 \\
\hline$S M R($ top $/$ mid $)[-]$ & 0.96 & 0.97 & 0.97 & 0.97 \\
\hline $\operatorname{SMR}($ top/bot $)[-]$ & 1.17 & 1.18 & 1.25 & 1.26 \\
\hline$S M R($ mid/bot $)[-]$ & 1.21 & 1.22 & 1.30 & 1.31 \\
\hline
\end{tabular}

Table 4 shows values of $J_{\text {sc,subcell }}^{\text {conc }}$ between 7.0 and $7.7 \mathrm{~A} / \mathrm{cm}^{2}$ for all the units and subcells. However, the current limiting subcell varies depending on the HCPV unit. The top subcell is limiting current in the SILO-Pyramid and DCCPC HCPV units, whereas the bot subcell is limiting current in the RTP and Trumpet ones. The optical polychromatic efficiency is greater than $80 \%$ for all the HCPV units, specifically between $81.0 \%$ (Trumpet HCPV unit) and $83.5 \%$ (DCCPC HCPV unit). Moreover, $\eta_{\text {opt }}$ is slightly higher in the top-currentlimiting HCPV units than in the bot-current-limiting ones. This last result may be related to the light absorption in the PMMA SOEs within the bot-wavelength region due to the longer optical path way (greater SOE height) of concentrated rays in the RTP and Trumpet SOEs compared to the other units. These $\eta_{\text {opt }}$ results can be compared to those when not considering most of the nonidealities in the optical simulations like: angular and spectral distribution of sunrays, light absorption within POE and SOE and spectral response of the solar cell (just like a perfect absorber). When using a monochromatic source of rays, in this case with a wavelength of $546 \mathrm{~nm}$, the optical efficiency of the HCPV units is simulated to be higher than when considering all those nonidealities, reaching a maximum of $86.6 \%$ (Trumpet HCPV unit). These highlight the importance of considering those nonidealities in order to have realistic simulated results of the optical elements designed.

In relation to the $S M R$ values under normal alignment, these are globally in the range between around 0.96 and 1.31 . Specifically, SMR(top/mid) is between 0.96 and 0.97 for the four HCPV units. Considering SMR(top/bot) values, these are near 1.17 for the SILOPyramid and DCCPC HCPV units, and around 1.25 for the RTP and Trumpet ones. SMR(top/bot) values higher for RTP and Trumpet HCPV units are in concordance with the bot subcell currentlimitation of both HCPV units. About $S M R$ (mid/bot) values, these are near 1.21 for SILO-Pyramid and DCCPC HCPV units and around 1.30 for RTP and Trumpet ones. Taking into account all the SMR values of all the HCPV units, those of the SILO-Pyramid unit are closer to 1, i.e., this HCPV unit concentrates sunrays with the lowest impact on the spectrum in relation to a typical TJ solar cell. This can be related to the lowest SOE height and absence of TIR. On the other hand, the Trumpet HCPV unit corresponds to the case of highest spectral change of concentrated sunrays respect to the standard spectrum. Note that, for all these HCPV units, $S M R=1$ is not achieved in none of the three versions, so the reference spectrum is distorted by effect of the optical system.

Ray tracing simulations are also conducted under different misalignment angles of the HCPV concentrator unit respect to the incident sunrays. It is worthy to analyze the corresponding simulation results under $1^{\circ}$ of tilt angle, which are summarized in Table 5.

Table 5 shows values of $J_{\text {sc,subcell }}^{\text {conc }}$ between around 6.3 and $7.7 \mathrm{~A} /$ $\mathrm{cm}^{2}$ for all the HCPV units and subcells under $1^{\circ}$ of tilt angle. For this tilt angle, it is found that the bot subcell is limiting the current generation in all the HCPV units except for the SILO-Pyramid one (with top subcell current limitation). The optical polychromatic efficiency is between $72.0 \%$ (Trumpet HCPV unit) and $78.2 \%$ (SILO-Pyramid HCPV unit). This is a reduction of $7 \%$ in average in comparison with normal alignment, being DCCPC and Trumpet HCPV units those with highest decrease (around 8\%), whereas for SILO-Pyramid and RTP HCPV units it is lowest (around 5\%). Note that, for this tilt angle, the top subcell is limiting current only in the SILO-Pyramid HCPV unit and the bot subcell is limiting current in the rest of the cases, whereas under normal alignment, top subcell is limiting in SILO-Pyramid and DCCPC HCPV units. Again, when not considering the non-idealities, the $\eta_{\text {opt }}$ increases, being in this case in the range between around 76\% (Trumpet HCPV unit) and $84 \%$ (DCCPC HCPV unit).

Considering the $S M R$ values under $1^{\circ}$ of misalignment, they are found to range between 0.90 and 1.34 . More in detail, SMR(top/ mid) is between around 0.90 (SILO-Pyramid HCPV unit) and 0.93 (Trumpet HCPV unit). This corresponds to a similar effect described for normal alignment but intensified. In the case of the DCCPC HCPV unit, SMR(top/mid) corresponds to around 0.98 , which is a value very close to 1 , i.e., near to equivalent conditions to the standard spectrum. SMR(top/bot) values are in the range from around 1.09 to 1.27 , being the lowest value that of the SILO-Pyramid HCPV unit. Finally, SMR(mid/bot) values range from around 1.21 (SILOPyramid HCPV unit) to 1.34 (RTP HCPV unit). Comparing to the

Table 5

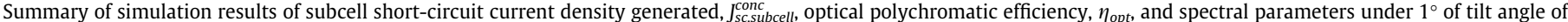
each HCPV unit respect to the sunrays for each Fresnel-based concentrator unit.

\begin{tabular}{|c|c|c|c|c|}
\hline Parameter & SILO-Pyramid & DCCPC & RTP & Trumpet \\
\hline$J_{s c, \text { top }}^{c o n c}\left[\mathrm{~A} / \mathrm{cm}^{2}\right]$ & 6.84 & 6.84 & 6.96 & 6.36 \\
\hline$J_{s c, \text { mid }}^{c c o n c}\left[\mathrm{~A} / \mathrm{cm}^{2}\right]$ & 7.60 & 7.03 & 7.34 & 6.86 \\
\hline$J_{s c, b o t}^{c o n c}\left[\mathrm{~A} / \mathrm{cm}^{2}\right]$ & 7.69 & 6.68 & 6.70 & 6.30 \\
\hline Limiting subcell & Top & Bot & Bot & Bot \\
\hline$\eta_{\text {opt }}[\%]$ & 78.2 & 76.3 & 76.6 & 72.0 \\
\hline$\eta_{\text {opt }}[\%]$ ideal case & 80.0 & 83.7 & 81.5 & 75.9 \\
\hline SMR(top/mid) $[-]$ & 0.90 & 0.98 & 0.95 & 0.93 \\
\hline$S M R(t o p / b o t)[-]$ & 1.09 & 1.26 & 1.27 & 1.24 \\
\hline$S M R($ mid/bot $)[-]$ & 1.21 & 1.28 & 1.34 & 1.33 \\
\hline
\end{tabular}


case under normal alignment, on one hand, SMR(top/mid) and SMR (mid/bot) for $1^{\circ}$ of tilt angle are further from the value equal to 1 . On the other hand, SMR(top/bot) is much closer to 1 for the SILOPyramid HCPV unit, whereas it separates from 1 in the rest of the HCPV units.

\subsubsection{Results under misalignment angles}

Simulated $J_{s c, \text { subcell }}^{\text {conc }}$ values adding the misalignment are shown in Fig. 6. The subcell that is limiting the current generated is that of the minimum $J_{s c, \text { subcell }}^{\text {conc }}$ value for each tilt angle. It can be seen, on one hand, top subcell current-limitation in the case of the SILOPyramid HCPV unit for all the misalignment angle range except beyond around $1.8^{\circ}$. It is also relative easy to observe the continuous bot subcell current limitation in the case of the RTP HCPV unit. There is also bot subcell current limitation for the whole tilt angle range in the case of the Trumpet HCPV unit. On the other hand, for the DCCPC HCPV unit there is an alternation between the limiting subcells, i.e., there is top subcell current limitation from normal alignment to around $0.8^{\circ}$ of tilt angle and from $1.2^{\circ}$ and beyond, but there is bot subcell current limitation in the interval between $0.8^{\circ}$ and $1.2^{\circ}$.

In order to analyze those spectral differences among the four HCPV units, the three SMR plots, (top/mid), (top/bot) and (mid/ bot), for each HCPV unit varying the misalignment angle are shown in Fig. 7. These plots present significant differences from each other not only under normal alignment but also under the different tilt angles. On one hand, it can be seen that $S M R($ top/mid) is lower than 1 (with a minimum of 0.87 at $1.2^{\circ}$ for the SILO-Pyramid HCPV unit) in all the cases except for tilt angles greater than around $1.8^{\circ}$ for the SILO-Pyramid HCPV unit and Trumpet one. Moreover, it shows a value within 0.87 and 1.09 , and is stable in general. On the other
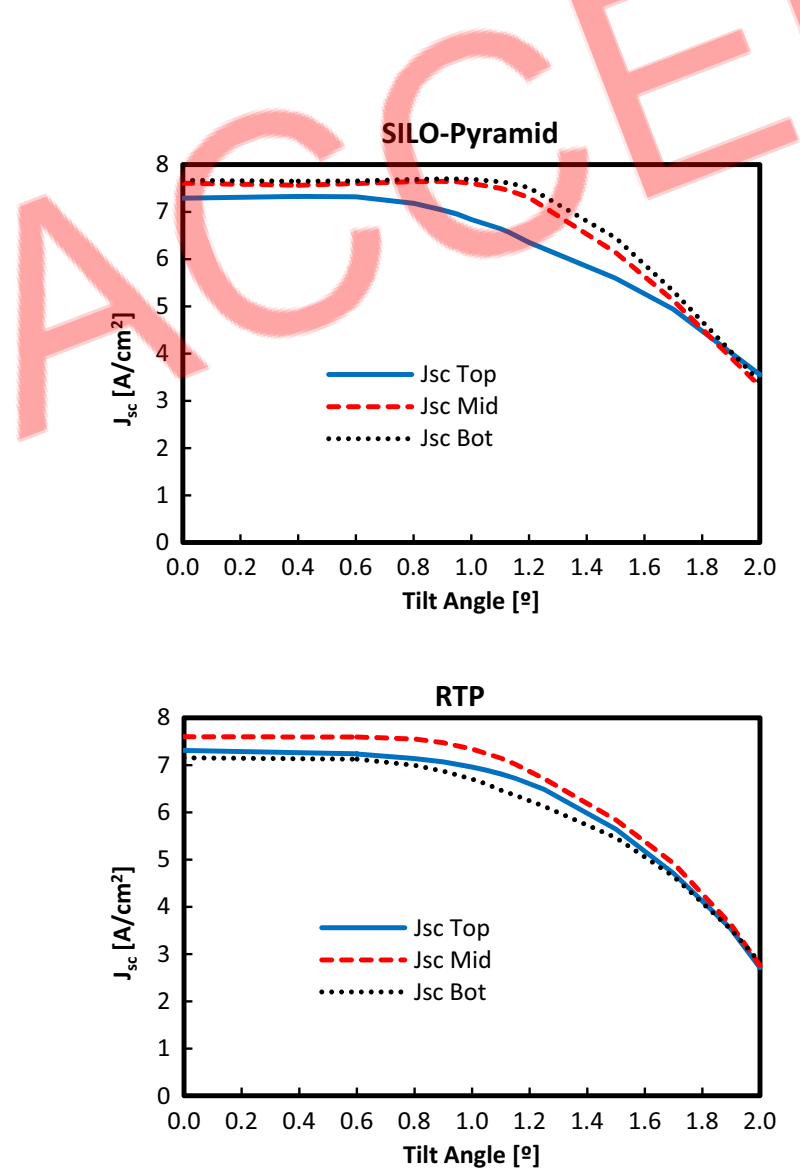

hand, $S M R($ top $/$ bot $)$ and $S M R($ mid/bot) present a relative higher variation with the tilt angle. For instance, in the case of the DCCPC HCPV unit, these last both SMR parameter curves have a peak maximum (with $S M R($ mid/bot $)=1.29$ ) at around $1.05^{\circ}$ and then they decrease rapidly for increased tilt angles until a value of 0.85 at $2.0^{\circ}$, whereas $S M R$ (top/mid) is maintained relative constant (0.96). Something similar occurs in the RTP HCPV unit but with the relative $S M R$ maximum (with $S M R($ mid/bot) $=1.34$ ) of the three parameters at around $1.1-1.2^{\circ}$, while the relative $S M R$ minimum for $S M R$ (top/bot) and $S M R$ (mid/bot) is of 1.19 at $2.0^{\circ}$. For this system, $S M R($ top $/ \mathrm{mid})$ is again relative constant (0.97). Considering the cases of the SILO-Pyramid and DCCPC HCPV units, the SMR parameter curves change relatively more strongly. In the case of the SILO-Pyramid HCPV unit SMR(top/mid) and SMR(top/bot) present a strong minimum (with $\operatorname{SMR}($ top $/$ mid $)=0.87$ ) at around $1.2^{\circ}$, whereas $S M R(\mathrm{mid} / \mathrm{bot})$ is relatively constant near a value of 1.2. As seen in Fig. 7, in terms of the spectral variations, the Trumpet HCPV unit, and especially the RTP one, present lowest dependency with the tilt angle.

Since the $\eta_{\text {opt }}$ values are also calculated including misalignments, it allows for a comparison between all the HCPV units with the different SOEs, including the case of having no SOE (see Fig. 8), to be carried out. This last case is more efficient for normal alignment, since there are no Fresnel losses involved. Nevertheless, its optical efficiency drops relative fast compared to the case of including a SOE. For instance, the $\eta_{\text {opt }}$ of the system without SOE is approximately $0 \%$ at around $1.2^{\circ}$, while it is kept within $70-$ $80 \%$ for the systems with SOE. The SILO-Pyramid represents the less sensitive SOE design to misalignment for angles between $1.7^{\circ}$ and $2.0^{\circ}$ among the HCPV units. In general, all these SOE designs present relative similar misalignment performance, although their angular characteristics spread each other for angles
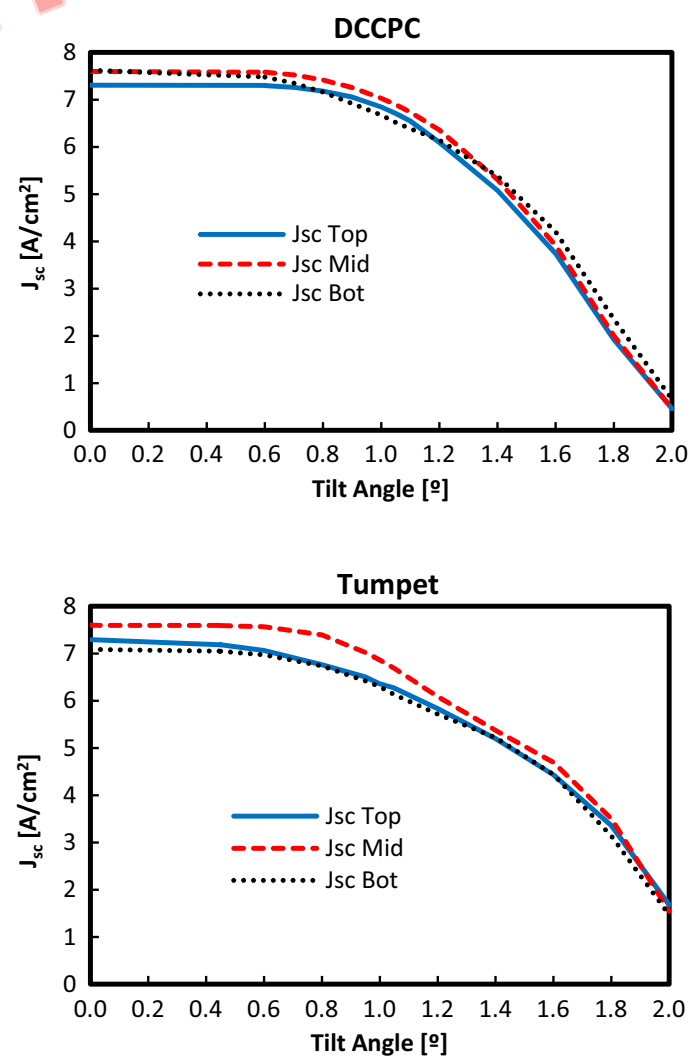

Fig. 6. $J_{s c, \text { subcell }}^{\text {conc }}$ values of the four Fresnel-based HCPV units for normal alignment and their evolution under the correspondent tilt angle. 

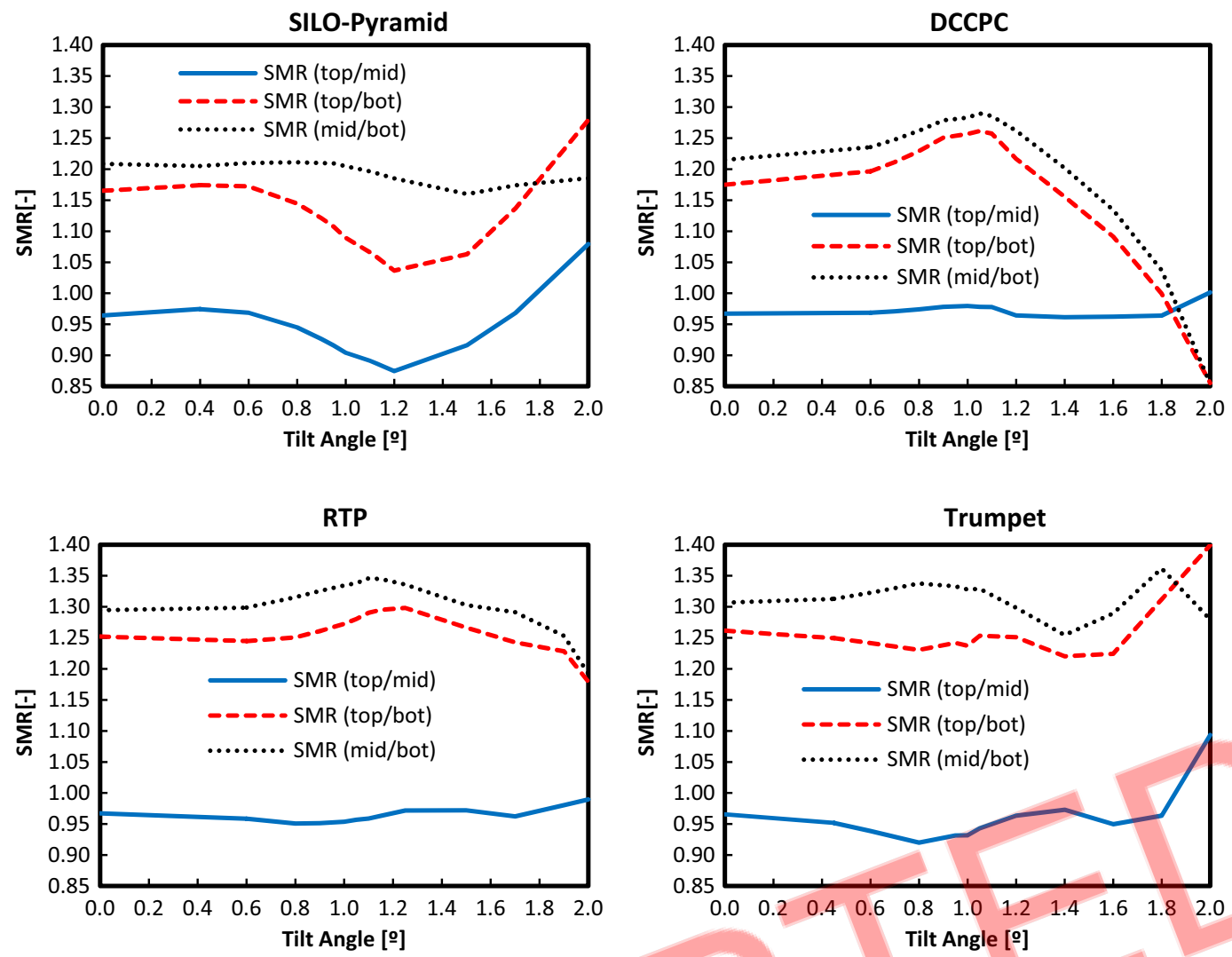

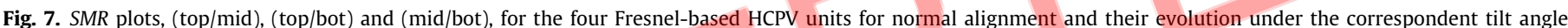
respect to the incident sunrays.

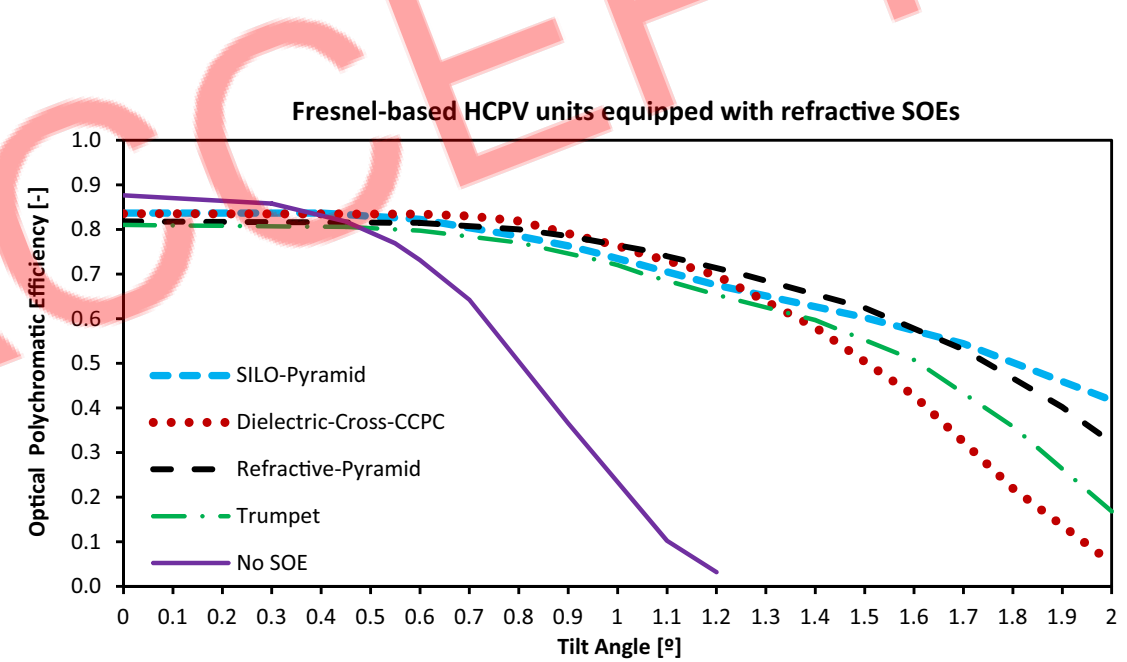

Fig. 8. Optical efficiency versus tilt angle for the four Fresnel-based HCPV units and also including the case of having no SOE (i.e., only POE and TJ solar cell).

Table 6

Summary of main parameters and simulation results.

\begin{tabular}{|c|c|c|c|c|c|}
\hline Parameter & No SOE & SILO-Pyramid & DCCPC & RTP & Trumpet \\
\hline Optical Polychromatic Efficiency [\%] & 87.7 & 83.4 & 83.6 & 81.8 & 81.0 \\
\hline Optical Polychr. Efficiency with AR [\%] & - & 85.5 & 87.1 & 83.5 & 82.4 \\
\hline Acceptance Angle $\left[{ }^{\circ}\right]$ & \pm 0.50 & \pm 1.13 & \pm 1.03 & \pm 1.11 & \pm 0.96 \\
\hline$C A P^{*}$ & 0.21 & 0.47 & 0.43 & 0.46 & 0.40 \\
\hline Opt. Polychr. Efficiency at Acceptance Angle [\%] & 78.9 & 75.3 & 75.2 & 73.6 & 72.9 \\
\hline SOE Volume $\left[\mathrm{mm}^{3}\right]$ & - & 916 & 871 & 1361 & 1020 \\
\hline SOE Height $[\mathrm{mm}]$ & - & 10.5 & 11.0 & 17.0 & 18.0 \\
\hline
\end{tabular}


greater than $1.4^{\circ}$. It can be seen that the DCCPC HCPV unit presents variation after $1.4^{\circ}$ misalignment.

Table 6 summarizes the values of all parameters for each HCPV unit, and also including the case of having no SOE for comparison reasons. The optical polychromatic efficiency is highest for the HCPV unit with the smallest SOE in terms of optical path length (SILO-Pyramid HCPV unit), with values slightly greater than $83 \%$, which is due to the relative lowest light absorption inside PMMA. The opposite case, with optical efficiency lower than $82 \%$, occurs for the SOE designs of more height: RTP and Trumpet HCPV units. For these two last cases, the bot subcell is limiting the current generated. The effective acceptance angle values of these designs range from $\pm 0.96^{\circ}$ (Trumpet HCPV unit) to $\pm 1.13^{\circ}$ (SILO-Pyramid HCPV unit). In terms of the effective concentration-acceptance angle product, $C A P^{*}$, the best SOE designs correspond to the SILOPyramid and the DCCPC HCPV units, with values 0.47 and 0.46 respectively. Applying perfect antireflective (AR) coating on the SOE entrance leads to better efficiency results. These improved optical polychromatic efficiencies range from 82.4\% (Trumpet HCPV unit) to a maximum of $87.1 \%$ (DCCPC HCPV unit). Obviously, simulating SOEs made of glass (e.g. BK7) and without considering any light absorption inside the materials, may lead to higher performance values. The volume of each SOE in each HCPV unit is also included, since the cost of manufacturing is related to it (Benitez et al., 2010). The RTP SOE has the relative highest material consume, with $1361 \mathrm{~mm}^{3}$. It is also remarkable the relative reduced volume of the SILO-Pyramid SOE with $871 \mathrm{~mm}^{3}$.

\subsubsection{Uncertainties}

The parameters defining both Fresnel lens and TJ solar cell are inherently subject to uncertainties. For example, in a real case, the dimensions of the lens, its focal distance, etc. are measured with a determined uncertainty. In order to analyze the impact of these uncertainties in the simulation results, optical simulations have been conducted with changed dimensions parameters. In this way, POE dimensions, focal length, facet spacing and also solar cell size have been varied and the correspondent $J_{\text {sc,subcell }}$, $\eta_{\text {opt }}$ and $S M R$ values obtained after optical simulation.

The variation of Fresnel lens parameter with highest impact on the results is its area, with variation in the $J_{\text {sc,subcell }}$ lower than $0.11 \mathrm{~A} / \mathrm{cm}^{2}$, lower than $0.23 \%$ in $\eta_{\text {opt }}$ and lower than 0.01 in $S M R$. Considering the variation in the TJ solar cell area, concentrated rays would be lost, since the exit surface is defined by that of the SOE. Therefore, a higher reduction of the $\eta_{\text {opt }}$ is expected, and it results to be lower than $6.2 \%$. Nevertheless, $J_{\text {sc,subcell }}$ and $S M R$ variations are expected to be lower than $0.4 \mathrm{~A} / \mathrm{cm}^{2}$ and 0.03 respectively.

Hence, according to the results above, no substantial changes in the simulation results may be expected as a consequence of the inherent uncertainties related to the simulation parameters.

\subsection{Qualitative modeling results}

Instead of analyzing the irradiance distributions over the TJ solar cell, short-circuit current density generated, $J_{\text {sc,subcell, }}^{\text {conc }}$ distributions are obtained and analyzed. The normalized $J_{s c, s u b c e l l}^{\text {conc }}$ spatial

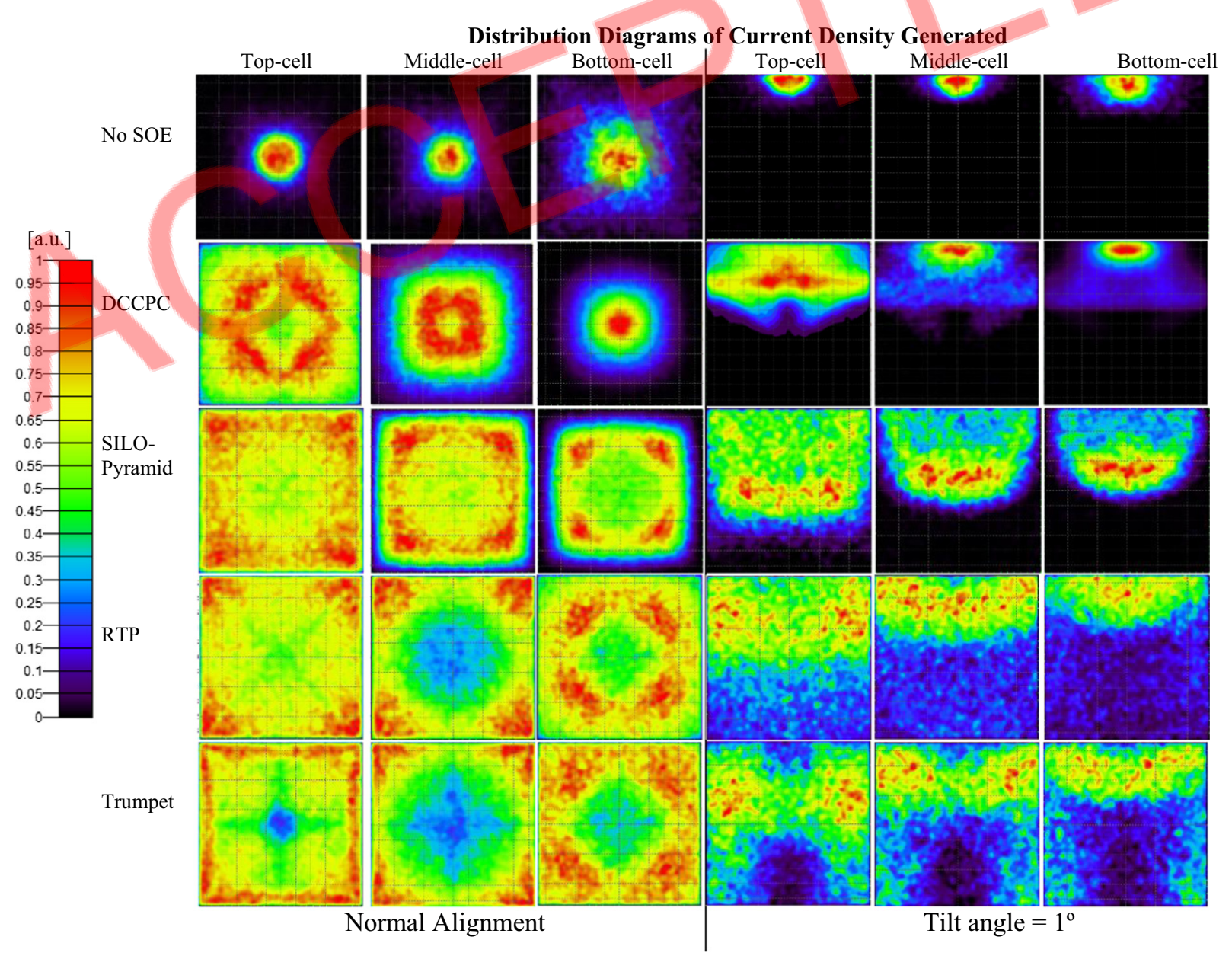

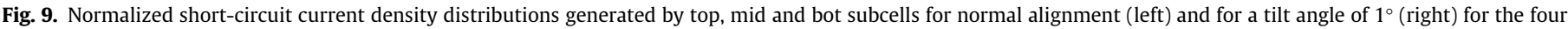
Fresnel-based HCPV units and, also for the case of the HCPV unit without any SOE. 


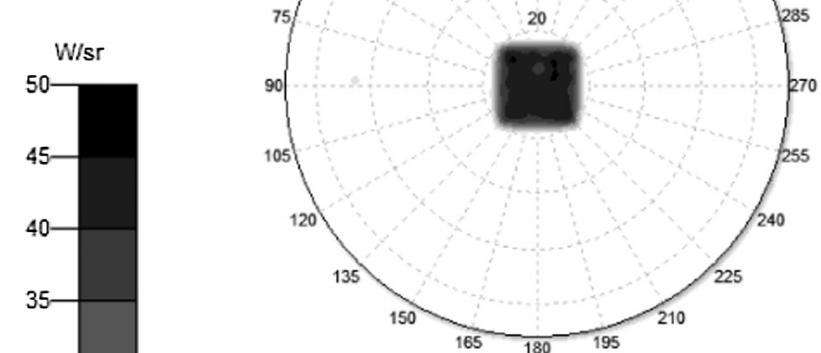

(a)

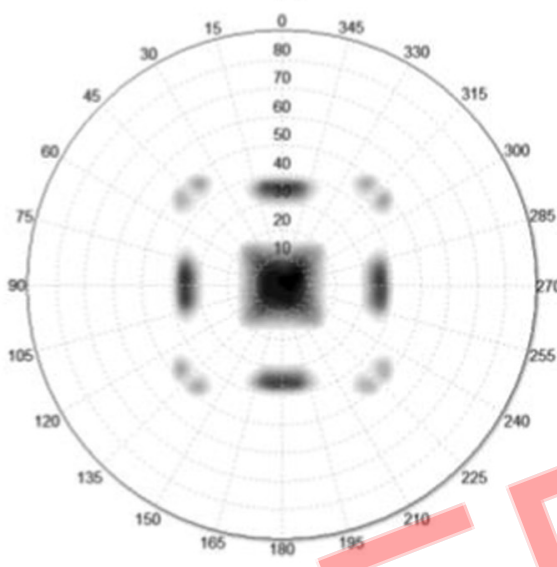

(c)

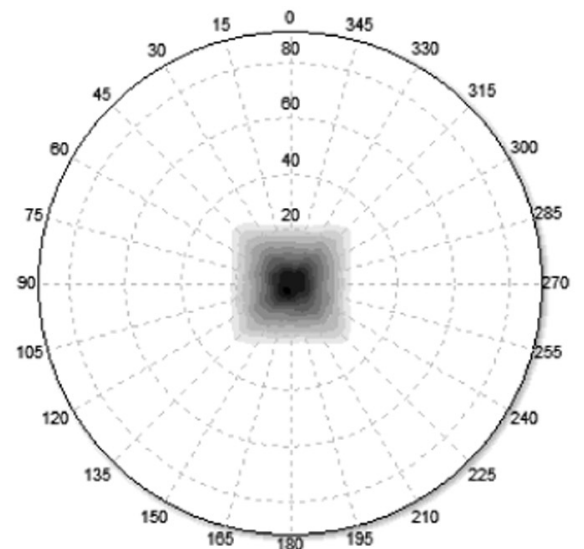

(b)

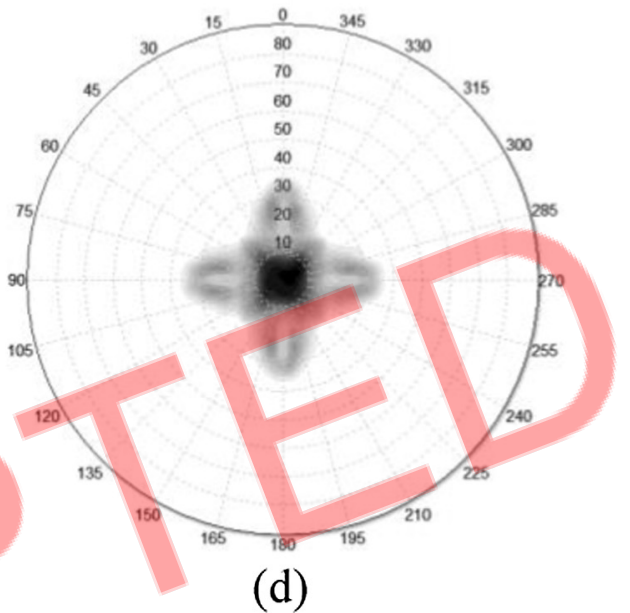

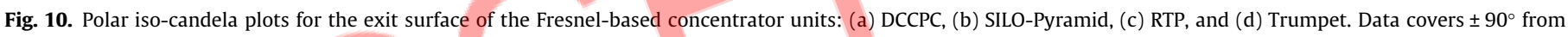
normal to the surface.

distributions for each subcell and for both normal alignment and $1^{\circ}$ of tilt angle are shown in Fig. 9 (Baig et al., 2012). Under normal alignment, most of SOE designs for the HCPV units lead to relatively uniform current density distributions as a consequence of rays mixing after TIR on side walls or after refraction on the convex entrance shape in the case of the SILO-based design. The lack of uniformity of the $J_{s c, b o t}^{c o n c}$ distribution in the DCCPC HCPV unit can be observed, this is due to a less interaction with the SOE walls for the rays in the region of the bot cell spectral response. Under $1^{\circ}$ of tilted angle, RTP and Trumpet HCPV units are the least sensitive ones to misalignments. This may be due to the TIR effect combined to the higher height of these SOEs compared to the other ones. As from the global results and considering the $J_{s c \text {,subcell }}^{\text {conc }}$ distribution uniformity, the RTP SOE may have here the best performance although the highest amount of material as commented above.

Not strictly considered as an output of this modeling, since the subcells structure of the TJ solar cell is ignored in this case, the incident angle of rays impinging the solar cell can be however shown through different plots. Fig. 10 shows polar iso-candela plots for the exit surface of each SOE in watts per steradian under normal alignment. The case with less concentrated rays angle respect to the normal of solar cell is that of the SILO-Pyramid HCPV unit, with almost all the rays with less than $20^{\circ}$ of incident angle. Among the SOEs based on TIR the maximum incident angle increases due to the multiple reflections on the side walls up to around $50^{\circ}$, which is the case of the RTP HCPV unit. Those rays with more incident angle (e.g. with $50^{\circ}$ ) may be more difficulty absorbed by the $\mathrm{TJ}$ solar cell (García-Linares et al., 2014; Bunthof et al., 2017).

\section{Conclusions and future works}

We present a complete optical modeling procedure intended to improve the simulation of typical Fresnel-based high concentrator photovoltaic (HCPV) units equipped with a refractive secondary optical element (SOE) and a typical triple-junction (TJ) solar cell. The Fresnel lens (primary optical element, POE) and the SOE are simulated as made of PMMA although any other material can be applied in the modeling. This powerful modeling takes into account some non-idealities for the ray tracing simulations but specially, the wavelength dependency of key material properties are simulated, like: absorption coefficient of the optical materials and the spectral response of the TJ solar cell.

As a consequence of simulating the spectral response of the TJ solar cell, the current density generated by each subcell is also simulated. It allows to calculate the optical polychromatic efficiency, which takes into account the correspondent subcell current limitation. Moreover, the simulation of the current density generation of each subcell allows to determine which subcell is limiting the current generation and to calculate the expected spectral matching ratio (SMR) parameters among the three subcells. Plots of current density uniformity generated on each subcell are even provided by this modeling. 
Four different HCPV units with the same aspheric Fresnel POE and TJ solar cell, and with four different SOEs are simulated. The design of each SOE is the result of a trade-off between the optical polychromatic efficiency and the acceptance angle of the correspondent HCPV unit.

Among the main results, the HCPV units equipped with higher SOEs, i.e. RTP and Trumpet, exhibit bottom subcell current limitation and lower optical polychromatic efficiency which may be related to the spectral absorption within the PMMA material in the spectral region of the bottom subcell. However, for the other HCPV units with smaller SOEs, i.e. DCCPC and SILO-Pyramid, there is top subcell current limitation and higher optical polychromatic efficiency. Moreover, for a determined HCPV unit, like specifically in the case of the DCCPC SOE, the current limitation can vary among the composing subcells. Specifically, top subcell is limiting for normal alignment and until around $0.8^{\circ}$ of misalignment angle of the HCPV unit respect to the incident sunrays and from $1.2^{\circ}$ and $2^{\circ}$ angle; whereas between $0.8^{\circ}$ and $1.2^{\circ}$ angle, the bottom subcell is limiting the current generation. Concerning the spectral performance of the four HCPV units, that with the RTP SOE presents the lowest variation of $S M R$ with the misalignment angle. Considering extreme values, SMR(mid/bot) is around 1.30 for the HCPV units with RTP and Trumpet SOEs under normal alignment. It is remarkable that, under normal alignment, SMR is never equal to 1 in none of its three versions in none of the four HCPV units. About the optical polychromatic efficiency, $\eta_{\text {opt }}$, and its variation with the misalignment angle, all the HCPV units exhibit similar performance until $1.4^{\circ}$ of misalignment angle. They show $\eta_{\text {opt }}$ between 81.0\% (Trumpet HCPV unit) and 83.6\% (DCCPC HCPV unit) with acceptance angles between $\pm 0.96^{\circ}$ (Trumpet HCPV unit) and $\pm 1.13^{\circ}$ (SILO-Pyramid HCPV unit)-resulting the effective concentration-angle product between 0.40 and 0.47 . However, the HCPV unit equipped with the DCCPC SOE presents little variation after $1.4^{\circ}$ of misalignment angle. Taking into account the uniformity of the current generated by each subcell in each HCPV unit, the HCPV units with SOEs based on total internal reflection exhibit an apparent better current density uniformity for a misalignment angle of $1^{\circ}$.

For future works, the validation of this modeling has to be confirmed with experimental data. Moreover, this modeling can be extended to quantify the impact of the uniformity of the current density generated by each subcell in each HCPV unit (EspinetGonzález et al., 2012). The incident angle of rays and the quantification of its impact on the current generation of each subcell is to be modeled also in future works (García-Linares et al., 2014), like also other non-idealities such as light scattering on the surfaces or light leakage between SOE and TJ solar cell (Baig et al., 2015).

\section{Funding}

European Regional Development Fund (ERDF) and Spanish Economy Ministry (ENE2013-45242-R and ENE2016-78251-R); Universidad de Jaén (UJA) and Caja Rural de Jaén (UJA2015/07/01). Financial support provided by the Universidad de Jaén Doctoral School.

\section{Acknowledgments}

The authors thank Lambda Research Corporation for its donation of TracePro optical software.

\section{References}

Anon., n.d. IEC 62670-1:2013 Concentrator Photovoltaic (CPV) Modules and Assemblies Performance Testing - Part 1: Standard Conditions. s.1.:ISBN 9782-8322-1120-5.

Baig, H., Heasman, K., Mallick, T., 2012. Non-uniform illumination in concentrating solar cells. Renew. Sustain. Energy Rev. 16 (8), 5890-5909.

Baig, H., Sellami, N., Mallick, T., 2015. Trapping light escaping from the edges of the optical element in a concentrating photovoltaic system. Energy Convers. Manage. 80, 238-246.

Beadie, G. et al., 2015. Refractive index measurements of poly(methylmethacrylate) (PMMA) from 0.4-1.6 $\mu \mathrm{m}$. Appl. Opt. 54 (31).

Benitez, P. et al., 2010. High performance Fresnel-based photovoltaic concentrator. Opt. Express 18 (S1), A25-A40.

Bunthof, L. et al., 2017. The illumination angle dependency of CPV solar cell electrical performance. Sol. Energy 144, 166-174.

Chen, Y., Chiang, H., 2015. Design of the secondary optical elements for concentrated photovoltaic units with fresnel lenses. Appl. Sci. 5 (4), 770-786.

Cooper, T. et al., 2013. Performance of compound parabolic concentrators with polygonal apertures. Sol. Energy 95, 308-318.

Cruz-Silva, O., Jaramillo, O.A., Borunda, M., 2016. Full analytical formulation for dielectric totally internally reflecting concentrators designs and solar applications. Renew. Energy 101 (804-815).

Domínguez, C., Antón, G., Askins, S., 2013. Current-matching estimation for multijunction cells within a CPV module by means of component cells. Prog. Photovoltaics: Res. Appl. 21 (7), 1478-1488.

Espinet-González, P. et al., 2012. Triple-junction solar cell performance under Fresnel-based concentrators taking into account chromatic aberration and offaxis operation. AIP Conf. Proc. 1477, 81-84.

Fernández, E., Almonacid, F., Ruiz-Arias, J., Soria-Moya, A., 2014. Analysis of the spectral variations on the performance of high concentrator photovoltaic modules operating under different real climate conditions. Sol. Energy Mater. Sol. Cells 127, 179-187.

Fernández, E., Soria-Moya, A., Almonacid, F., Aguilera, J., 2016. Comparative assessment of the spectral impact on the energy yield of high concentrator and conventional photovoltaic technology. Sol. Energy Mater. Sol. Cells 147, 185-197.

Fu, L., Leutz, R., Annen, H., 2010. Secondary optics for Fresnel lens solar concentrators. Nonimaging Optics: Efficient Design for Illumination and Solar Concentration VII 7785, p. 778509-6.

García-Linares, P. et al., 2014. Effect of the encapsulant temperature on the angular and spectral response of multi-junction solar cells. IEEE Photovoltaic Spec. Conf. (PVSC), 3298-3303.

James, L., 1989. Use of imaging refractive secondaries in photovoltaic concentrators, Albuquerque, NM: SAND89-7029.

Kumar, V., Shrivastava, R., Untawale, S.P., 2015. Fresnel lens: a promising alternative of reflectors in concentrated solar power. Renew. Sustain. Energy Rev. 44, $376-$ 390.

Leutz, R., Suzuki, A., 2001. Nonimaging Fresnel Lenses. Springer-Verlag, s.l..

Miller, D., Kempe, M., Kennedy, C., Kurtz, S., 2011. Analysis of transmitted optical spectrum enabling accelerated testing of multijunction concentrating photovoltaic designs. Opt. Eng. 50 (1).

Miñano, J. et al., 2013. Free-form optics for Fresnel-lens-based photovoltaic concentrators. Opt. Express 21, A494-A502.

O'Gallagher, J., 2008. Nonimaging Optics in Solar Energy. Morgan \& Claypool Publishers, s.l.

Pérez-Higueras, P., Fernández, E., 2015. High Concentrator Photovoltaics: Fundamentals, Engineering and Power Plants. Springer International Publishing, s.l.

Reddy et al., 2014. Design and optimisation of elliptical hyperboloid concentrator with helical receiver. Sol. Energy 108, 515-524.

Rodrigo, P., Fernández, E., Almonacid, F., Pérez-Higueras, P., 2017. Quantification of the spectral coupling of atmosphere and photovoltaic system performance: indexes, methods and impact on energy harvesting. Sol. Energy Mater. Sol. Cells $163,73-90$.

Shanks, K. et al., 2017. Prototype fabrication and experimental investigation of a conjugate refractive reflective homogeniser in a cassegrain concentrator. Sol. Energy 142, 97-108.

Shanks, K. et al., 2016. Theoretical investigation considering manufacturing errors of a high concentrating photovoltaic of cassegrain design and its experimental validation. Sol. Energy 131, 235-245.

Victoria, M., Domínguez, C., Antón, I., Sala, G., 2009. Comparative analysis of different secondary optical elements for aspheric primary lenses. Opt. Express 17 (8), 6487-6492. 